\title{
On a Conjecture of Webb
}

\author{
Jacques Thévenaz \\ Institut de Mathématiques \\ Université de Lausanne \\ CH-1015 Lausanne \\ Switzerland
}

Let $p$ be a prime divisor of the order of a finite group $G$ and let $S_{p}(G)$ be the poset of non-trivial $p$-subgroups of $G$. Associated to this poset, we consider the simplicial complex $\Delta\left(S_{p}(G)\right)$ of chains of non-trivial $p$-subgroups, called Brown's complex (in honour of K.S. Brown who first introduced it). The group $G$ acts by conjugation on $S_{p}(G)$, hence also on $\Delta\left(S_{p}(G)\right)$, and this natural action of $G$ can be used for handling $p$-local information in the theory of $p$-modular representations and mod $p$ cohomology of the group $G$, as for instance in [W1], [W2], [W3], [KR], [Bo], [Th]. We refer to Webb's survey paper [W2] for more details about Brown's complex and applications.

In connection with these recent developments, P.J. Webb stated the following conjecture.

CONJECTURE (Webb [W2, 4.2]). Let $\left|\Delta\left(S_{p}(G)\right)\right|$ be the geometric realization of the simplicial complex $\Delta\left(S_{p}(G)\right)$. Then the orbit space $\left|\Delta\left(S_{p}(G)\right)\right| / G$ is contractible.

By passing to the barycentric subdivision of $\Delta\left(S_{p}(G)\right)$, one can view $\left|\Delta\left(S_{p}(G)\right)\right| / G$ as the geometric realization of the simplicial complex associated with a suitable poset. Thus we can again work with a poset.

Webb first realized that the Euler characteristic of $\left|\Delta\left(S_{p}(G)\right)\right| / G$ is trivial [W1, 8.2] (see also [Th, 4.4] for another proof), and then more generally he proved that $\left|\Delta\left(S_{p}(G)\right)\right| / G$ is $\bmod p$ acyclic [W3, 2.6]. This is the main evidence for the conjecture. Note also that if $G$ is a group of Lie type in characteristic $p$, then $\Delta\left(S_{p}(G)\right)$ is $G$-homotopy equivalent to the Tits building of $G$ (see [TW, 2.4] for details), and the conjecture holds because the orbit complex of the building is a single simplex, hence contractible.

The purpose of this note is to prove the conjecture in a few cases, as follows.

THEOREM A. Webb's conjecture holds for a $p$-solvable group $G$. 
THEOREM B. Webb's conjecture holds if a Sylow $p$-subgroup of $G$ is either abelian, or generalized quaternion, or TI.

Recall that a Sylow $p$-subgroup $P$ is called $T I$ if $P^{g} \cap P=1$ for all $g \notin N_{G}(P)$. In other words every $p$-subgroup is contained in a unique Sylow $p$-subgroup.

Both theorems easily follow from rather elementary results on $p$-subgroups and control of fusion which we describe in the first section. The proofs appear in the second section.

\section{The poset of orbits}

The orbit set $\Delta\left(S_{p}(G)\right) / G$ is not a simplicial complex, because in general there are several orbits of simplices with given orbits of vertices. Thus we pass to the barycentric subdivision, that is, we consider the poset $\Sigma_{p}(G)$ of (non empty) chains in $S_{p}(G)$. The associated simplicial complex $\Delta\left(\Sigma_{p}(G)\right)$ consists of chains of chains of non-trivial $p$-subgroups, but we concentrate on the poset $\Sigma_{p}(G)$ itself. An element $\sigma \in \Sigma_{p}(G)$ will be written

$$
\sigma=\left(P_{0}<P_{1}<\ldots<P_{n}\right)
$$

where $P_{i} \in S_{p}(G)$ and $n=\operatorname{dim}(\sigma)$. It is immediate to check that the poset $\Sigma_{p}(G)$ satisfies the crucial condition :

$$
\tau \leq \sigma \quad \text { and } \quad \tau^{g} \leq \sigma \quad \text { imply that } \quad \tau=\tau^{g} \quad\left(\tau, \sigma \in \Sigma_{p}(G), g \in G\right)
$$

Thus $\Sigma_{p}(G)$ is a regular $G$-poset in the sense of [CR, §66].

Now write $[\sigma]_{G}$ for the orbit of $\sigma$ under $G$. The orbit set $\Sigma_{p}(G) / G$ is a poset, with the order relation $[\tau]_{G} \leq[\sigma]_{G}$ provided that $\tau^{\prime} \leq \sigma^{\prime}$ for some $\tau^{\prime} \in[\tau]_{G}$ and $\sigma^{\prime} \in[\sigma]_{G}$. An easy consequence of the regularity condition (1.1) (see [CR, 66.6] for details) is that $\Delta\left(\Sigma_{p}(G)\right) / G$ is a simplicial complex and is isomorphic to $\Delta\left(\Sigma_{p}(G) / G\right)$, the simplicial complex associated with the poset $\Sigma_{p}(G) / G$. Moreover by $\left[\mathrm{CR}, 66.1\right.$ and 66.8], the orbit space $\left|\Delta\left(S_{p}(G)\right)\right| / G$ (which we are interested in) is homeomorphic to the space associated with the poset $\Sigma_{p}(G) / G$. Thus we only have to work with this poset.

Let $f: H \rightarrow G$ be a homomorphism of finite groups. Since $S_{p}(G)$ and $\Sigma_{p}(G)$ do not involve the trivial subgroup, $f$ can induce a map of posets $f_{*}: \Sigma_{p}(H) \rightarrow \Sigma_{p}(G)$ only if no $p$-subgroup is contained in $K=\operatorname{Ker}(f)$, that is, if $p$ does not divide $|K|$. If this condition is satisfied, it is clear that $f_{*}$ exists, is order preserving and preserves the length of chains of $p$-subgroups. Moreover if $\sigma, \tau \in \Sigma_{p}(H)$ are $H$-conjugate, then $f_{*}(\sigma)$ and $f_{*}(\tau)$ are $G$-conjugate, and therefore $f_{*}$ induces a map $\bar{f}_{*}: \Sigma_{p}(H) / H \rightarrow \Sigma_{p}(G) / G$ which is order preserving. 
(1.2) PROPOSITION. Let $K$ be a normal subgroup of $G$ of order prime to $p$ and let $Q=G / K$. Then the canonical group homomorphism $f: G \rightarrow Q$ induces an isomorphism of posets $\bar{f}_{*}: \Sigma_{p}(G) / G \rightarrow$ $\Sigma_{p}(Q) / Q$.

Proof. Let $\sigma=\left(P_{0}<\ldots<P_{n}\right) \in \Sigma_{p}(Q)$ and let $H$ be the inverse image of $P_{n}$ in $G$. Let $Q_{n}$ be a Sylow $p$-subgroup of $H$ (so that $H=K Q_{n}$ ). Then $f$ induces an isomorphism $Q_{n} \cong P_{n}$ and so there is a chain in $\Sigma_{p}(G)$ (with maximal element $Q_{n}$ ) mapping onto $\sigma$. It follows in particular that $\bar{f}_{*}: \Sigma_{p}(G) / G \rightarrow \Sigma_{p}(Q) / Q$ is surjective.

Let $\sigma=\left(P_{0}<\ldots<P_{n}\right) \in \Sigma_{p}(G)$ and $\tau=\left(Q_{0}<\ldots<Q_{n}\right) \in \Sigma_{p}(G)$. Assume that $[f(\sigma)]_{Q}=$ $[f(\tau)]_{Q}$, so that there exists $g \in G$ with $f(\sigma)=f(\tau)^{f(g)}$. We have to show that $[\sigma]_{G}=[\tau]_{G}$, so replacing $\tau$ by $\tau^{g}$, we can assume that $f(\sigma)=f(\tau)$. Then $f\left(P_{n}\right)=f\left(Q_{n}\right)$, that is, $K P_{n}=K Q_{n}$. Since $P_{n}$ and $Q_{n}$ are Sylow $p$-subgroups of $K P_{n}$, there exists $k \in K$ such that $P_{n}=Q_{n}^{k}$. Replacing $\tau$ by $\tau^{k}$, we can assume further that $P_{n}=Q_{n}$. Now $f$ induces an isomorphism $P_{n} \cong f\left(P_{n}\right)$ and the images of the subgroups $P_{i}$ and $Q_{i}$ are equal. Therefore $P_{i}=Q_{i}$ for all $i$, and $\sigma=\tau$. This proves the injectivity of $\bar{f}_{*}$.

If $f: H \rightarrow G$ is a group homomorphism, the proposition reduces the study of the induced map $\bar{f}_{*}: \Sigma_{p}(H) / H \rightarrow \Sigma_{p}(G) / G$ to the case of the inclusion $\operatorname{Im}(f) \rightarrow G$. Thus we can assume that $f$ is an inclusion. Recall that a subgroup $H$ of $G$ controls $p$-fusion in $G$ if some Sylow $p$-subgroup of $G$ is contained in $H$ (i.e. $|G: H|$ is prime to $p$ ) and the following condition is satisfied: whenever $Q$ is a $p$-subgroup of $H$ and $Q^{g} \leq H$ for some $g \in G$, then $g=c h$ with $c \in C_{G}(Q)$ and $h \in H$. Here $C_{G}(Q)$ denotes the centralizer of $Q$ in $G$.

(1.3) PROPOSITION. Let $H$ be a subgroup of $G$ which controls $p$-fusion in $G$. Then the inclusion $i: H \rightarrow G$ induces an isomorphism of posets $\bar{i}_{*}: \Sigma_{p}(H) / H \rightarrow \Sigma_{p}(G) / G$.

Proof. The surjectivity is easy: if $P_{n}$ is the maximal subgroup of $\sigma \in \Sigma_{p}(G)$, then since $|G: H|$ is prime to $p$, there exists $g \in G$ such that $P_{n}^{g} \leq H$; then $\sigma^{g} \in \Sigma_{p}(H)$ and $\bar{i}_{*}\left(\left[\sigma^{g}\right]_{H}\right)=\left[\sigma^{g}\right]_{G}=[\sigma]_{G}$, as required.

Now let $\sigma, \tau \in \Sigma_{p}(H)$ such that $[\sigma]_{G}=[\tau]_{G}$, that is, $\tau=\sigma^{g}$ for some $g \in G$. Let $P_{n}$ be the maximal subgroup of $\sigma$. Since $P_{n}^{g}$ is the maximal subgroup of $\tau$ which is a chain in $H$, we have $P_{n}^{g} \leq H$. By control of fusion, it follows that $g=c h$ with $c \in C_{G}\left(P_{n}\right)$ and $h \in H$. Since $c$ stabilizes the whole chain $\sigma$, we obtain $\tau=\sigma^{h}$, proving the injectivity of $\bar{i}_{*}$.

(1.4) REMARK. It is possible to express the results above in a categorical setting as in $[\mathrm{Pu}]$, where Puig introduces the Frobenius category. We also introduce a weak form of the Frobenius category. Let $\operatorname{Frob}_{p}(G) \quad$ (respectively $\operatorname{Weak}_{p}(G)$ ) be the category whose objects are all $p$-subgroups of $G$ (including 1) and whose set of morphisms between the $p$-subgroups $P$ and $Q$ is equal to $C_{G}(P) \backslash T_{G}(P, Q)$ (respectively $N_{G}(P) \backslash T_{G}(P, Q)$ ), where $T_{G}(P, Q)=\left\{g \in G \mid P^{g} \leq Q\right\}$. Similarly we define $\operatorname{CFrob}_{p}(G)$ (respectively $\operatorname{CWeak}_{p}(G)$ ) to be the category whose objects are all chains of $p$-subgroups of $G$ (which may include 1) and whose set of morphisms between the chains $\sigma$ and $\tau$ is equal to $C_{G}(\sigma) \backslash T_{G}(\sigma, \tau)$ (respectively $\left.N_{G}(\sigma) \backslash T_{G}(\sigma, \tau)\right)$, where $T_{G}(\sigma, \tau)$ is defined as follows: if $\sigma=\left(P_{0}<\ldots<P_{n}\right)$ and $\tau=\left(Q_{0}<\ldots<Q_{m}\right)$, then $T_{G}(\sigma, \tau)$ is the set of all $g \in G$ such that $P_{i}^{g} \leq Q_{\phi(i)}$ for all $i$, for some order-preserving injective map $\phi:\{0, \ldots, n\} \rightarrow\{0, \ldots, m\}$. 
Given a group homomorphism $f: H \rightarrow G$, we want to understand when $f$ induces an equivalence of categories $f_{*}: \operatorname{Frob}_{p}(H) \stackrel{\sim}{\rightarrow} \operatorname{Frob}_{p}(G)$, and similarly for the other categories. It is not hard to prove that $f_{*}: \operatorname{CWeak}_{p}(H) \stackrel{\sim}{\rightarrow} \operatorname{CWeak}_{p}(G)$ if and only if the map of posets $\bar{f}_{*}: \Sigma_{p}(H) / H \rightarrow \Sigma_{p}(G) / G$ is an isomorphism. On the other hand it is well-known that $f_{*}: \operatorname{Frob}_{p}(H) \stackrel{\sim}{\rightarrow} \operatorname{Frob}_{p}(G)$ if and only if $p$ does not divide $|\operatorname{Ker}(f)|$ and $\operatorname{Im}(f)$ controls $p$-fusion in $G$. Therefore the two propositions above say that

$$
f_{*}: \operatorname{Frob}_{p}(H) \stackrel{\sim}{\rightarrow} \operatorname{Frob}_{p}(G), \quad \Longrightarrow \quad f_{*}: \operatorname{CWeak}_{p}(H) \stackrel{\sim}{\rightarrow} \operatorname{CWeak}_{p}(G)
$$

For completeness, we also mention that

$$
f_{*}: \operatorname{Frob}_{p}(H) \stackrel{\sim}{\rightarrow} \operatorname{Frob}_{p}(G) \quad \Longleftrightarrow \quad f_{*}: \operatorname{CFrob}_{p}(H) \stackrel{\sim}{\rightarrow} \operatorname{CFrob}_{p}(G),
$$

and that

$$
f_{*}: \operatorname{CWeak}_{p}(H) \stackrel{\sim}{\rightarrow} \operatorname{CWeak}_{p}(G) \quad \Longrightarrow \quad f_{*}: \operatorname{Weak}_{p}(H) \stackrel{\sim}{\rightarrow} \operatorname{Weak}_{p}(G) \text {. }
$$

We do not elaborate further on this. Indeed only $\operatorname{Frob}_{p}(G)$ seems to play an important role in group theory, for instance in questions of classification (see $[\mathrm{Pu}]$ ) and in group cohomology (see [Mi]).

\section{Webb's conjecture}

We start with the well-known observation that Webb's conjecture holds if $O_{p}(G) \neq 1$. As usual, $O_{p}(G)$ denotes the largest normal $p$-subgroup of $G$.

(2.1) LEMMA. If $P=O_{p}(G)$ is non-trivial, then $\Sigma_{p}(G) / G$ is contractible.

Proof. It is here more convenient to work with $S_{p}(G)$. By Section $1,\left|\Delta\left(\Sigma_{p}(G) / G\right)\right|$ is homeomorphic to $\left|\Delta\left(S_{p}(G)\right)\right| / G$. Now the orbit space $X / G$ of a $G$-space $X$ is contractible if $X$ is $G$-contractible, because a $G$-equivariant homotopy $F: X \times[0,1] \rightarrow X$ between $i d_{X}$ and the constant map (onto a $G$ fixed point) induces a homotopy $F: X / G \times[0,1] \rightarrow X / G$. Thus it suffices to prove that $\left|\Delta\left(S_{p}(G)\right)\right|$ is $G$-contractible. Quillen [Qu, 2.4] proved that it is contractible via the maps

$$
Q \mapsto Q P \mapsto P \quad\left(Q \in S_{p}(G)\right)
$$

and this contraction is obviously $G$-equivariant (see also [TW, 1.2]).

Our strategy is now simply to use the results of Section 1 to reduce to the situation where $O_{p}(G) \neq 1$. We first consider Theorem A of the introduction.

(2.2) PROPOSITION. If $G$ is $p$-solvable, then $\Sigma_{p}(G) / G$ is contractible.

Proof. Let $K=O_{p^{\prime}}(G)$, the largest normal subgroup of $G$ of order prime to $p$, and let $Q=G / K$. By Proposition 1.2, $\Sigma_{p}(G) / G$ is isomorphic to $\Sigma_{p}(Q) / Q$. But by definition of a $p$-solvable group, the group $Q$ has a non-trivial normal $p$-subgroup. Therefore by Lemma $2.1, \Sigma_{p}(Q) / Q$ is contractible.

Next we prove Theorem B of the introduction. 
(2.3) PROPOSITION. Let $P$ be a Sylow $p$-subgroup of $G$ and assume that $P$ is either abelian, or generalized quaternion, or TI. Then $\Sigma_{p}(G) / G$ is contractible.

Proof. If $P$ is abelian, then it was already known to Burnside that $H=N_{G}(P)$ controls $p$-fusion in $G$. Therefore by Proposition $1.3, \Sigma_{p}(G) / G \cong \Sigma_{p}(H) / H$ and the latter is contractible by Lemma 2.1. For the reader's convenience, we recall Burnside's argument. If $Q, Q^{g} \leq N_{G}(P)$, then $Q, Q^{g} \leq P$, and so $Q \leq P$ and $Q \leq P^{g^{-1}}$. Since $P$ is abelian, $P$ and $P^{g^{-1}}$ are Sylow $p$-subgroups of $C_{G}(Q)$, hence conjugate by some $c \in C_{G}(Q)$. It follows that $g^{-1} c \in N_{G}(P)$, as required.

If $P$ is a generalized quaternion 2-group, then $P$ has a unique subgroup $Z$ of order 2. But $N_{G}(Z)$ controls 2 -fusion in $G$ and we conclude again by Proposition 1.3 and Lemma 2.1. We also recall why $N_{G}(Z)$ controls 2 -fusion. If $Q, Q^{g} \leq N_{G}(Z)$, then $Z, Z^{g} \leq N_{G}(Z)$. But since $Z$ is the unique subgroup of $N_{G}(Z)$ of order 2 , we have $Z=Z^{g}$, hence $g \in N_{G}(Z)$.

If $P$ is $T I$, then $N_{G}(P)$ controls $p$-fusion in $G$ and we conclude again by Proposition 1.3 and Lemma 2.1. The proof that $N_{G}(P)$ controls fusion is a straightforward consequence of the fact that every $p$-subgroup $Q$ is contained in a unique Sylow $p$-subgroup.

REMARKS. (a) The Brauer-Suzuki theorem on groups $G$ with a generalized quaternion Sylow 2subgroup asserts that $G / O_{p^{\prime}}(G)$ has a central subgroup of order 2. Thus the result above is also a consequence of Proposition 1.2.

(b) It is clear that the argument of Proposition 2.3 says that if $G$ has a subgroup $H$ which controls $p$-fusion and which has a normal $p$-subgroup, then $\Sigma_{p}(G) / G$ is contractible.

We end this paper with another application of Proposition 1.3.

(2.4) PROPOSITION. If Webb's conjecture (for the prime $p$ ) holds for the symmetric group $S_{p n}$, then it also holds for each symmetric group $S_{p n+k}$ where $0 \leq k \leq p-1$.

Proof. It is well-known that $S_{m-1}$ controls $p$-fusion in $S_{m}$ if $p$ does not divide $m$. The proof is left to the reader. Repeated applications of this property lead to the result, thanks to Proposition 1.3. 
References

[Bo] S. Bouc, Projecteurs dans l'anneau de Burnside, projecteurs dans l'anneau de Green, modules de Steinberg généralisés, J. Algebra, to appear.

[CR] C.W. Curtis, I. Reiner, Methods of Representation Theory, Volume II, Wiley-Interscience, New-York, 1987.

[KR] R. Knörr, G.R. Robinson, Some remarks on a conjecture of Alperin, J. London Math. Soc. 39 (1989), 48-60.

[Mi] G. Mislin, Group homomorphisms inducing isomorphisms in mod-p cohomology, Comment. Math. Helv., to appear.

[Pu] L. Puig, La classification des groupes finis simples: bref aperçu et quelques conséquences internes, Astérisque 92-93 (1982), 101-128.

[Qu] D. Quillen, Homotopy properties of the poset of non-trivial p-subgroups of a group, Adv. in Math. 28 (1978), 101-128.

[Th] J. Thévenaz, Locally determined functions and Alperin's conjecture, preprint, 1990.

[TW] J. Thévenaz, P.J. Webb, Homotopy equivalence of posets with a group action, J. Combin. Theory Ser. A, to appear.

[W1] P.J. Webb, A local method in group cohomology, Comment. Math. Helv. 62 (1987), 135-167.

[W2] P.J. Webb, Subgroup complexes, Proc. Symp. Pure Math. 47 (1987), 349-365.

[W3] P.J. Webb, A split exact sequence of Mackey functors, preprint, 1989.

July 1990 